\title{
Review of Ocular Injuries in Patients with Orbital Wall Fractures: A 5-Year Retrospective Analysis
}

This article was published in the following Dove Press journal: Clinical Ophthalmology

\author{
Stephanie B Terrill' \\ Hyelin You ${ }^{2}$ \\ Heidi Eiseman ${ }^{\prime}$ \\ Michael E Rauser (D) ' \\ 'Department of Ophthalmology, Loma \\ Linda University Eye Institute, Loma \\ Linda, CA, USA; ${ }^{2}$ Department of \\ Ophthalmology, Loma Linda University \\ School of Medicine, Loma Linda, \\ CA, USA
}

Purpose: The purpose of this study was to determine the incidence of minor and major ocular injuries in patients with orbital wall fractures at Loma Linda University Health, a level-one trauma center, to help determine the most appropriate setting for the initial dilated fundus examination by ophthalmologists.

Methods: A retrospective study was performed from January 2008 to January 2013 of patients diagnosed with orbital wall fracture secondary to trauma. Exclusion criteria included unknown mechanism of injury, the absence of ophthalmology consultation, or absence of imaging. Data collected included age, gender, mechanism of injury, visual acuity, and anterior/ posterior segment findings. Ocular injuries were categorized as either minor or major.

Results: Of 567 charts reviewed, 460 met criteria and were included for analysis. In the analysis, $86.5 \%$ of patients were male, and $81.3 \%$ were Caucasian. The most common mechanism of orbital fracture was blunt injury. Visual acuity was better than 20/100 in $82.4 \%$ of patients. On chart review, $81.1 \%$ of patients were found to have either a minor injury, a major injury, or both. The most common injury was subconjunctival hemorrhage (53.5\%). Globe rupture $(2.9 \%)$ and vision-threatening posterior segment findings such as retinal tear and choroidal rupture $(1.3 \%)$ were relatively rare. Only one retinal detachment $(0.2 \%)$ was found, specifically in the setting of severe injury with concomitant globe rupture.

Conclusion: Knowledge of the common ocular injuries associated with orbital fractures will help emergency department (ED) physicians and ophthalmologists provide the dilated fundus exam in the most appropriate setting. The most frequent injuries identified were non-vision threatening, and visually significant posterior segment findings were relatively rare $(1.3 \%)$. Thus, for the majority of patients presenting to the ED with orbital fracture, a dilated fundus exam can be performed at a later date in the outpatient clinic setting, unless urgent orbital fracture surgery is planned.

Keywords: ocular trauma, orbital wall fracture, subconjunctival hemorrhage, globe rupture, entrapment, vision loss

\section{Introduction}

Orbital fractures secondary to trauma are often complicated by ocular injuries. ${ }^{1-3}$ As such, consultations for a comprehensive ophthalmic exam are routine in private as well as academic sectors. A comprehensive ocular examination is necessary to prevent lasting visual and functional deficits, though the timing of the initial exam is debatable. Patients with orbital fractures are routinely assessed on an outpatient basis initially and, as demonstrated by Mellema et al, visually asymptomatic patients with orbital fractures are unlikely to have sustained severe injuries. ${ }^{4}$ Nonetheless, serious ocular findings such as globe rupture or entrapment still warrant an emergency department (ED) consultation by the ophthalmology service.
Correspondence: Michael E Rauser Loma Linda University Eye Institute, I 1370 Anderson Street, Suite 1800, Loma Linda, CA 92354, USA

Tel + I 9095582112

Email mrauser@llu.edu 
Identifying the most common associations between orbital fractures and ocular pathologies, such as visionthreatening retinal injuries, will aid in the management and appropriate timing of a dilated fundus exam by ophthalmologists. This paper highlights the incidence of the most common ocular injuries sustained by patients with orbital fractures at Loma Linda University Health, a level-one trauma facility.

\section{Methods}

A retrospective review was performed of patients with orbital fractures evaluated at Loma Linda University Health, a level-one trauma center. Between January 2008 and January 2013, 567 charts of patients with orbital fractures seen in the ED were reviewed. Exclusion criteria included the absence of an ophthalmology consult, absence of a CT scan read, or unknown mechanism of injury. Maxillofacial CT scan images were not reviewed personally by the investigators. A fracture of any orbital wall was included, but not classified further based on location. All fractures were due to trauma and categorized as blunt (representing physical assault), motor vehicle accidents (MVA), fall, or gunshot wound (GSW).

Data collected from the written ophthalmology consult included age, gender, mechanism of injury, visual acuity, and anterior/posterior segment findings. Ocular injuries were further separated into major and minor categories. A minor ocular injury was defined as an injury unlikely to result in permanent vision loss: subconjunctival hemorrhage, limitation of extraocular movement, pupil abnormality, commotion retinae, corneal abrasion, proptosis, chemosis, traumatic iritis, decreased sensation, retinal hemorrhage, macular hemorrhage (without foveal or choroidal involvement), iridodialysis, and enophthalmos. A major ocular injury was defined as an injury likely to cause permanent visual impairment: hyphema, retrobulbar hemorrhage, ruptured globe, disc edema, entrapment, choroidal rupture, partial thickness corneal laceration, and retinal tear. The incidence of major and minor injuries in the setting of orbital fractures is reported.

The study received approval by the LLU Institutional Review Board, adhered to the standards of Declaration of Helsinki, and was Health Insurance Portability and Accountability Act compliant. As this study was performed retrospectively, the requirement for consent was waived by the LLU Institutional Review Board.

\section{Results}

Between January 2008 and January 2013, charts of 567 patients with orbital fractures seen in the ED were reviewed. Exclusion criteria included the absence of an ophthalmology consultation ( $\mathrm{n}=75)$, absence of the maxillofacial CT scan read $(n=29)$, and unknown mechanism of injury ( $\mathrm{n}=3$ ). Thus, of the 567 charts reviewed, 460 were included for analysis. As observed in previous series, there was a strong male predominance at $86.5 \%$. Other demographics such as ethnicity, age, and type of injury are noted in Table 1. Blunt trauma was the most common mechanism of injury in men while falls accounted for the highest number in women (Figure 1).

The patients' visual acuity was assessed using a Snellen near card, though visual acuity could not be obtained in 103 of the 460 patients (22.4\%) on initial ED presentation due to altered mental status, intubation, critical patient condition, and lack of documentation. Of the patients with documented visual acuity on initial presentation $(n=357)$, vision was better than $20 / 100$ in $82.4 \%$ of patients and equal to or greater than $20 / 30$ in $44.8 \%$ of patients. Only 63 patients or $17.6 \%$ of the total charts reviewed had visual acuity of equal to or less than 20/ 100. Of these 63 patients, $19.0 \%(n=12)$ had sustained a globe rupture, while the remaining $81.0 \%$ of patients had

Table I Patient Demographics

\begin{tabular}{|l|l|}
\hline Demographics & N (\%) \\
\hline $\begin{array}{l}\text { Sex } \\
\text { Male }\end{array}$ & $398(86.5 \%)$ \\
Female & $122(13.5 \%)$ \\
\hline Ethnicity & \\
White & $374(81.3 \%)$ \\
Black & $40(8.7 \%)$ \\
Other & $33(7.2 \%)$ \\
Asian & $11(2.4 \%)$ \\
Hispanic & $1(0.2 \%)$ \\
\hline Type of Injury & \\
Blunt & $222(48.3 \%)$ \\
MVA & $117(25.4 \%)$ \\
Fall & $112(24.3 \%)$ \\
GSW & $9(2.0 \%)$ \\
\hline Age & Years \\
Range & $0-95$ \\
Median \pm SD & $32 \pm 20.7$ \\
\hline
\end{tabular}

Abbreviations: MVA, motor vehicle accident; GSW, gunshot wound. 


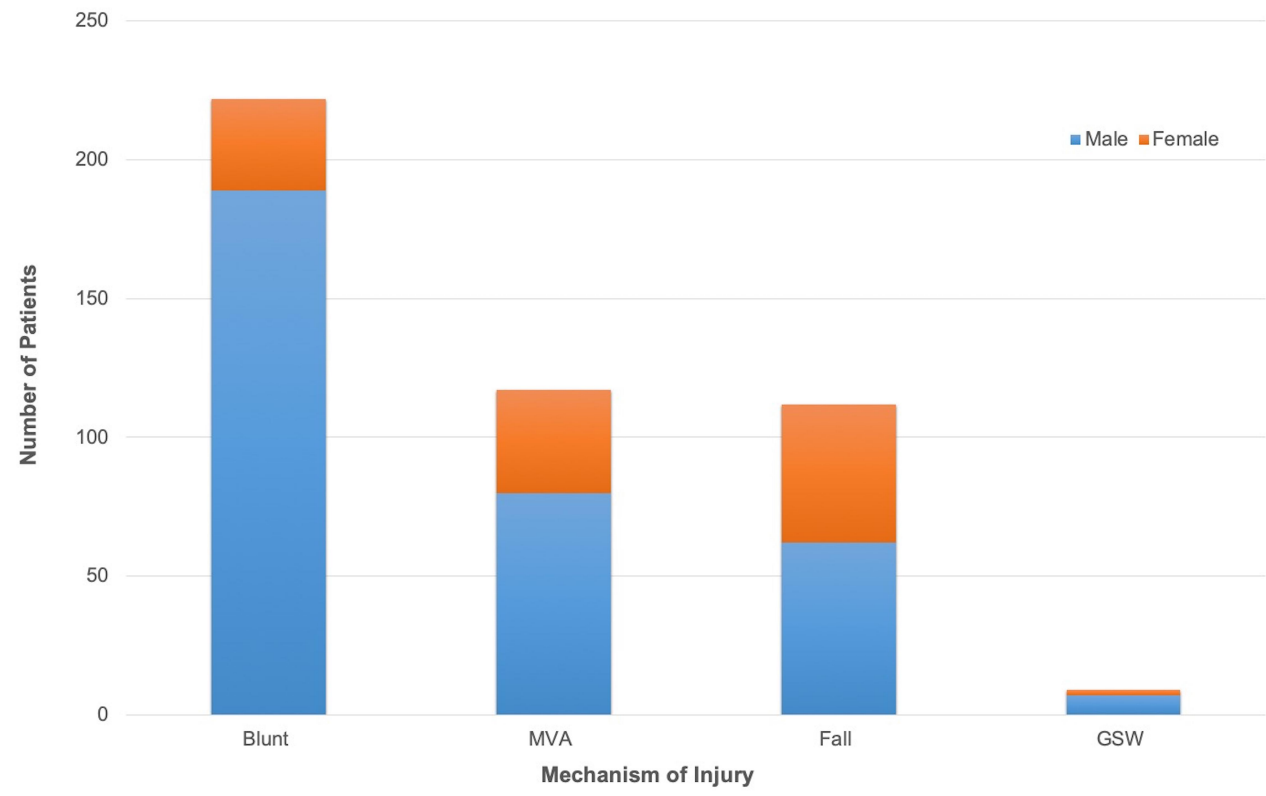

Figure I Mechanism of injury.

Abbreviations: MVA, motor vehicle accident; GSW, gunshot wound.

incurred various injuries without any notable trends in incidence of injury type.

Overall, $81.1 \%$ of the patients $(n=373)$ included were found to have sustained either a minor injury, a major injury, or both. There were 299 patients $(65.0 \%)$ with at least one minor injury and 74 patients $(16.1 \%)$ with at least one major injury. Among these individuals, a total of 765 injuries were noted. There were 679 minor injuries identified, representing $88.8 \%$ of all documented ocular injuries, which were unlikely to result in permanent visual impairment. By far, subconjunctival hemorrhage $(\mathrm{n}=246)$ and limitation of extraocular movement $(n=185)$ were the most common minor injuries (Table 2).

There were 86 major injuries identified, representing $11.2 \%$ of all documented injuries, with hyphema $(n=23)$ and retrobulbar hemorrhage $(\mathrm{n}=16)$ being the most prevalent (Table 3). Thirteen ruptured globes were identified $(2.9 \%)$, and a visual acuity of $20 / 800$ or worse was found in 12 of the 13 eyes. Of the 13 globe ruptures, there were no associated retinal complications noted on initial ED evaluation. However, when seven of these patients were further evaluated in later clinic visits, one patient was found to have developed a retinal detachment on two-week follow-up. Entrapment, confirmed by forcedduction testing, was identified in 7 of 460 patients (1.5\%). Six of the seven patients who had restriction of extraocular muscles and were $\leq 21$ years old. Only one out of the seven entrapped patients endorsed diplopia.
Of the identified major injuries, there was a relatively low incidence of major retinal complications, such as choroidal rupture and retinal tear $(\mathrm{n}=6 ; 1.3 \%)$. Specifically, there were five choroidal ruptures $(1.1 \%)$ and one retinal tear $(0.2 \%)$ on initial presentation. The patient with retinal tear subsequently underwent successful laser retinopexy. No treatment was provided for the patients with choroidal rupture. Additionally, there were no patients who presented with retinal detachment on initial ED evaluation in our study, though of the patients who had at least one follow-up clinic visit $(n=109$; average

Table 2 Incidence of Minor Ocular Injuries (\%)

\begin{tabular}{|l|l|}
\hline Injury Type & Rate (\%) \\
\hline Subconjunctival hemorrhage & $53.5 \%$ \\
Limitation of extraocular movement & $40.2 \%$ \\
Pupil abnormality & $17.8 \%$ \\
Commotio retinae & $13.8 \%$ \\
Corneal abrasion & $8.3 \%$ \\
Proptosis & $6.3 \%$ \\
Chemosis & $2.8 \%$ \\
Traumatic iritis & $2.0 \%$ \\
Decreased sensation & $1.1 \%$ \\
Retinal hemorrhage & $2.4 \%$ \\
Macular hemorrhage & $0.9 \%$ \\
Iridodialysis & $0.4 \%$ \\
Enophthalmos & $0.2 \%$ \\
\hline
\end{tabular}

Note: ${ }^{a}$ Incidence of injury found in all patients reviewed, $n=460$. 
Table 3 Incidence of Major Ocular Injuries (\%)

\begin{tabular}{|l|l|}
\hline Injury Type & Rate (\%) $^{\mathbf{a}}$ \\
\hline Hyphema & $5.0 \%$ \\
Retrobulbar hemorrhage & $3.5 \%$ \\
Ruptured globe & $2.9 \%$ \\
Disc edema & $1.7 \%$ \\
Entrapment & $1.5 \%$ \\
Choroidal rupture & $1.1 \%$ \\
Partial thickness corneal laceration & $0.9 \%$ \\
Retinal tear & $0.2 \%$ \\
\hline
\end{tabular}

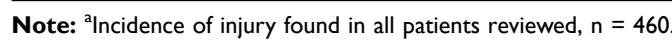

time to follow-up=26.1 days), there was one patient who had developed a retinal detachment within two weeks of a ruptured globe as mentioned previously. This patient subsequently received retinal detachment repair.

\section{Discussion}

It is known that serious ocular injuries associated with orbital fractures have the potential to impair vision if not properly recognized and treated in a timely manner. ${ }^{5,6}$ Because the incidence of major injuries varies in the literature (10\% to $27.6 \%$ ), it is important that co-managing specialties are aware of the most common minor and major injuries sustained by patients with orbital fractures.7,8 This knowledge could facilitate the delivery of timeefficient care in the appropriate consult setting by the ophthalmologist, by aiding in the determination of patient acuity and the need for evaluation in the ED versus outpatient follow-up.

Recent studies have reported that patients with penetrating trauma, visual acuity deficits, afferent pupillary defects, and posterior orbital fractures were at highest risk for vision loss. ${ }^{8,9}$ Additionally, Richani et al identified four signs and symptoms to be predictors of severityblurred vision, pain with eye movement, poor visual acuity, and restricted mobility — with the presence of two or more of these signs being predictive of severe ocular trauma, indicating the need for urgent ophthalmologic consultation. ${ }^{10}$ This is consistent with the results of our study which demonstrates that visual acuity deficits can be an indicator of potential serious ocular injury as 12 of the $13(92.3 \%)$ patients with ruptured globes were found to have VA of 20/800 or worse. For co-managing specialists, poor visual acuity or vision loss unexplained by anterior segment examination may indicate concomitant visionthreatening complications, though correlation with an eye examination and CT imaging is required. Patients with orbital fractures who are sedated, unconscious, or otherwise unable to provide an accurate visual acuity should be examined by an ophthalmologist.

With respect to the mechanism of injury, a prospective study by al-Qurainy et al found assault to be the highest percentage (49.9\%), while falls and road traffic accidents accounted for $19 \%$ and $12.4 \%$, respectively - values comparable to a more recent study performed by Chow et al (48\%, 17\%, and $21 \%$, respectively). ${ }^{5,9}$ Conversely, Amrith et al reported road traffic accidents to account for the largest proportion, at $36.5 \%$, while the percentage for assault was $12.5 \%{ }^{11}$ In this study, blunt trauma was found to be the cause of $48.3 \%$ of cases while the percentage of falls and motor vehicle accidents were found to be $24.3 \%$ and $25.4 \%$, respectively. Given the variability among several studies of adequate sample size, the differences observed are likely secondary to regional and cultural factors.

Similar to previous reports, our study found anterior segment abnormalities and limitation of extraocular movement to be the most common injuries reported overall. ${ }^{7,12-}$ ${ }^{15}$ By far, subconjunctival hemorrhage was the most prevalent anterior segment finding in orbital fracture patients. However, while subconjunctival hemorrhages, due to their appearance, have the potential to alarm co-managing subspecialists, they are not vision-threatening unless there is concern for concomitant globe rupture, such as in cases with 360-degree involvement.

Diplopia (double vision) is a symptom that concerns patients and can be a "red-flag" to co-managing surgical subspecialists. Evaluation for entrapment is a common reason for which ophthalmologists are consulted on patients with orbital fractures. Entrapment of extraocular muscles is more prevalent in the pediatric population, as demonstrated by our study where six of the seven patients with entrapment were $\leq 21$ years old. The increased prevalence of entrapment in children and young adults may be explained by a phenomenon termed "trap door fracture". First described by Soll and Poley, this is a fracture where a transient increase in orbital pressure results in a temporary displacement of bone which immediately recoils back to its original position, allowing for extraocular muscles and/or orbital tissue to become entrapped in the process. ${ }^{16}$ The increased bone elasticity in the younger population likely facilitates entrapment by this mechanism. ${ }^{17,18}$ It should be noted, however, that while entrapment requires urgent surgery preferably within 48 hours, diplopia due to impingement is not an ophthalmologic emergency and surgery 
should be deferred until it can be performed by an adept surgeon. ${ }^{19,20}$

In this study, we found vision-threatening injuries to account for $11.9 \%$ of all ocular injuries that presented to the ED. Of these major injuries, posterior segment findings such as retinal tear or choroidal rupture encompassed a small percentage $(1.3 \%)$ of all injuries. Of these, only one condition, the retinal tear, was treatable by laser retinopexy. Additionally, during the five-year duration of our study, there was only one case of retinal detachment $(0.2 \%)$, identified after initial examination in a patient who had suffered a globe rupture two weeks prior, who subsequently received treatment for the retinal detachment. The low incidence of retinal detachments in this study is consistent with the relatively low reported values in the literature regarding retinal detachments associated with orbital fractures, ranging from $0.7 \%$ to $2.9 \%$. $^{6,8}$ Such a low incidence should give ED providers confidence that the dilated fundus examination to evaluate the posterior segment can be reasonably deferred at the initial patient encounter to the outpatient clinic setting for the majority of patients, typically within one to two weeks. This delay also allows time for the reduction of pain and periorbital swelling, permitting a more comfortable and comprehensive examination of the patient. If urgent orbital fracture surgery is undertaken, then a dilated fundus exam should be considered prior to the planned surgery.

Furthermore, there is evidence that orbital fractures in the setting of ocular trauma may counterintuitively play a protective role by providing decompression of the orbital tissue as compared with ocular trauma without accompanying fracture. ${ }^{21,22} \mathrm{We}$, therefore, recommend that ED providers prioritize the assessment of visual acuity, anterior segment findings, extraocular muscle function, and imaging, instead of a dilated fundus examination, in patients presenting with traumatic orbital wall fractures. However, in patients for whom a dilated fundus examination is deferred, it is important to provide counseling on the warning symptoms of a retinal tear or detachmentnew onset of significant photopsias, floaters, curtain-like shadow, and visual field loss - that may develop prior to their scheduled outpatient appointment. Patients who develop such symptoms should be instructed to contact their ophthalmologist to be examined urgently.

\section{Limitations}

This study has several limitations. Because we retrospectively examined patients who had been seen on an emergent basis immediately following the ocular trauma, baseline visual function and anterior/posterior segment exam findings were unavailable for the majority of individuals. Additionally, while visually threatening ocular injuries after orbital fracture was uncommon on initial evaluation, it is difficult to determine the exact incidence of permanent vision loss that may have developed in patients with injuries originally classified as minor ocular trauma, as most patients did not have follow-up visits with our institution. Lastly, the patient demographic of this study was confined to the region surrounding one medical facility and thus may not be generalizable to populations of significantly different cultural or socioeconomic background. However, though several theoretical limitations exist in the generalizability of our findings, our results are in concordance with the consensus of current literature regarding this issue.

\section{Conclusion}

For specialists involved in the co-management of orbital fracture patients, it is reasonable to consult ophthalmology when there is a notable decrease in visual acuity, vision loss not explained by anterior segment examination, evidence of anterior segment trauma or ruptured globe, in the setting of penetrating injury, when the patient is sedated/ unconscious, or when the patient is unable to participate in a basic bedside eye examination performed by the primary provider. However, for the majority of patients presenting to the ED with orbital fracture, a dilated fundus examination can be performed at a later date in the outpatient clinic setting, unless urgent orbital fracture surgery is planned.

\section{Acknowledgments}

The authors acknowledge the support of Loma Linda University, Loma Linda, CA, USA.

\section{Disclosure}

The authors report no conflicts of interest in this work. The abstract of this paper was presented at the ARVO Annual Meeting as a poster presentation/conference talk with interim findings. The poster's abstract was published in ARVO Annual Meeting Abstracts in Investigative Ophthalmology \& Visual Science: https://iovs.arvojour nals.org/article.aspx?articleid=2268143.

\section{References}

1. Ho TQ, Jupiter D, Tsai JH, Czerwinski M. The incidence of ocular injuries in isolated orbital fractures. Ann Plast Surg. 2017;78(1):59-61. doi:10.1097/SAP.000000000000748 
2. Riaz N, Chatha AA, Warraich RA, Hanif S, Chinar KA, Khan SR. Ophthalmic injuries in orbito-zygomatic fractures. J Coll Physicians Surg Pak. 2014;24(9):649-652.

3. Barry C, Coyle M, Idrees Z, Dwyer MH, Kearns G. Ocular findings in patients with orbitozygomatic complex fractures: a retrospective study. J Oral Maxillofac Surg. 2008;66(5):888-892. doi:10.1016/j. joms.2008.01.005

4. Mellema PA, Dewan MA, Lee MS, Smith SD, Harrison AR. Incidence of ocular injury in visually asymptomatic orbital fractures. Ophthalmic Plast Reconstr Surg. 2009;25(4):306-308. doi:10.1097/ IOP.0b013e3181aa9a73

5. Al-Qurainy IA, Stassen LF, Dutton GN, Moos KF, el-Attar A. The characteristics of midfacial fractures and the association with ocular injury: a prospective study. Br J Oral Maxillofac Surg. 1991;29 (5):291-301. doi:10.1016/0266-4356(91)90114-K

6. Holt GR, Holt JE. Incidence of eye injuries in facial fractures: an analysis of 727 cases. Otolaryngol Head Neck Surg. 1983;91(3):276279. doi:10.1177/019459988309100313

7. Jamal BT, Pfahler SM, Lane KA, et al. Ophthalmic injuries in patients with zygomaticomaxillary complex fractures requiring surgical repair. J Oral Maxillofac Surg. 2009;67:986-989. doi:10.1016/j. joms.2008.12.035

8. Andrews BT, Jackson AS, Nazir N, Hromas A, Sokol JA, Thurston TE. Orbit fractures: identifying patient factors indicating high risk for ocular and periocular injury. Laryngoscope. 2016;126(Suppl 4):S5S11. doi:10.1002/lary.25805

9. Chow J, Parthasarathi K, Mehanna P, Whist E. Primary assessment of the patient with orbital fractures should include pupillary response and visual acuity changes to detect occult major ocular injuries. $J$ Oral Maxillofac Surg. 2018;76(11):2370-2375. doi:10.1016/j. joms.2018.04.024

10. Richani K, Do TH, Merritt HA, Pfeiffer ML, Chuang AZ, Phillips ME. Screening criteria for detecting severe ocular injuries in the setting of orbital fractures. Ophthalmic Plast Reconstr Surg. 2019;35(6):609-614. doi:10.1097/IOP.0000000000001422

11. Amrith S, Saw SM, Lim TC, Lee TK. Ophthalmic involvement in cranio-facial trauma. J Craniomaxillofac Surg. 2000;28(3):140-147. doi:10.1054/jcms.2000.0138
12. Roh JH, Jung JW, Chi M. A clinical analysis of bilateral orbital fracture. J Craniofac Surg. 2014;25(2):388-392. doi:10.1097/ SCS.0000000000000646

13. He D, Blomquist PH, Ellis E 3rd. Association between ocular injuries and internal orbital fractures. J Oral Maxillofac Surg. 2007;65 (4):713-720. doi:10.1016/j.joms.2006.09.006

14. Ross M, El-Haddad C, Deschênes J. Ocular injury in orbital fractures at a level I trauma center. Can J Ophthalmol. 2017;52(5):499-502. doi:10.1016/j.jcjo.2017.01.013

15. Cook T. Ocular and periocular injuries from orbital fractures. $J A m$ Coll Surg. 2002;195(6):831-834. doi:10.1016/S1072-7515(02) 01479-5

16. Soll DB, Poley BJ. Trapdoor variety of blowout fracture of the orbital floor. Am J Ophthalmol. 1965;60:269-272. doi:10.1016/0002-9394 (65)90927-X

17. Grant JH 3rd, Patrinely JR, Weiss AH, Kierney PC, Gruss JS. Trapdoor fracture of the orbit in a pediatric population. Plast Reconstr Surg. 2002;109(2):482-495.

18. Tse R, Allen L, Matic D. The white-eyed medial blowout fracture. Plast Reconstr Surg. 2007;119(1):277-286. doi:10.1097/01. prs.0000237032.59094.c2

19. Su Y, Shen Q, Bi X, Lin M, Fan X. Delayed surgical treatment of orbital trapdoor fracture in paediatric patients. $\mathrm{Br} J$ Ophthalmol. 2019;103(4):523-526. doi:10.1136/bjophthalmol-2018-311954

20. Alinasab B, Qureshi AR, Stjärne P. Prospective study on ocular motility limitation due to orbital muscle entrapment or impingement associated with orbital wall fracture. Injury. 2017;48(7):1408-1416. doi:10.1016/j.injury.2017.04.039

21. Noh H, Chung JK, Woo KI, Kim YD. Occurrence of ocular injury and orbital fracture in orbital blunt trauma patients at tertiary care center emergency room. Graefes Arch Clin Exp Ophthalmol. 2020. doi:10.1007/s00417-020-04786-6

22. Santamaria J, Mehta A, Reed D, Blegen H, Bishop B, Davies B. Orbital roof fractures as an indicator for concomitant ocular injury. Graefes Arch Clin Exp Ophthalmol. 2019;257(11):2541-2545. doi:10.1007/s00417-019-04455-3
Clinical Ophthalmology

\section{Publish your work in this journal}

Clinical Ophthalmology is an international, peer-reviewed journal covering all subspecialties within ophthalmology. Key topics include: Optometry; Visual science; Pharmacology and drug therapy in eye diseases; Basic Sciences; Primary and Secondary eye care; Patient Safety and Quality of Care Improvements. This journal is indexed on PubMed

\section{Dovepress}

Central and CAS, and is the official journal of The Society of Clinical Ophthalmology (SCO). The manuscript management system is completely online and includes a very quick and fair peer-review system, which is all easy to use. Visit http://www.dovepress.com/ testimonials.php to read real quotes from published authors. 